\title{
Multiple energy x-ray holography: Incident-radiation polarization effects
}

\author{
P. M. Len \\ Physics Department, University of California, Davis, California 95616 \\ T. Gog* \\ Oak Ridge National Laboratory at the National Synchrotron Light Source, Brookhaven National Laboratory, Upton, New York 11973 \\ D. Novikov \\ Hamburger Synchrotronstrahlungslabor HASYLAB am Deutsches Elektronen-Synchrotron DESY, 22603 Hamburg, Germany \\ R. A. Eisenhower \\ Oak Ridge National Laboratory at the National Synchrotron Light Source, Brookhaven National Laboratory, Upton, New York 11973 \\ G. Materlik \\ Hamburger Synchrotronstrahlungslabor HASYLAB am Deutsches Elektronen-Synchrotron DESY, 22603 Hamburg, Germany \\ C. S. Fadley \\ Physics Department, University of California, Davis, California 95616 \\ and Materials Sciences Division, Lawrence Berkeley National Laboratory, Berkeley, California 94720
}

(Received 21 November 1996; revised manuscript received 12 February 1997)

\begin{abstract}
Multiple energy $\mathrm{x}$-ray holography $(\mathrm{MEXH})$ measures both phase and amplitude information for $\mathrm{x}$ rays scattered from an incident reference beam, from which three-dimensional atomic images can be directly reconstructed. The angular distribution of the x-ray scattering is highly dependent on the polarization direction via the Thomson scattering cross section. We consider here the effect of incident $\mathrm{x}$-ray polarization on images of Fe atoms reconstructed from theoretical and experimental $\mathrm{MEXH}$ data for $\alpha-\mathrm{Fe}_{2} \mathrm{O}_{3}(001)$ (hematite). We also illustrate such polarization effects theoretically in the enhancement of specific atomic structural information of ideal Fe trimers, and a Ge $\delta$-layer buried in $\mathrm{Si}(001)$, where the use of different polarization modes and experimental geometries is found to strongly influence atomic images. [S0163-1829(97)04224-0]
\end{abstract}

\section{INTRODUCTION}

Gabor proposed holography as a means of surpassing the current limitations of electron microscopy, specifically by experimentally recording the amplitude and phase of scattered wave fronts relative to a direct unscattered reference wave. ${ }^{1}$ Later, Szöke observed that the phases of atomically scattered photoelectron or fluorescent $\mathrm{x}$-ray wave fronts can be referenced to the direct or unscattered wave front. ${ }^{2}$ This assumes that the electron or x-ray emission is from a highly localized core excitation. Thus, structural information on the atomic environment surrounding each emitting atom is uniquely encoded in the holographic photoelectron or fluorescence intensities, as detected in the far field outside the sample. Algorithms to retrieve direct three-dimensional atomic images from these holograms have subsequently been developed $^{3-7}$ and successfully implemented in application to e.g., photoelectron diffraction data. ${ }^{8-12}$

These holographic principles have also been demonstrated theoretically for $\mathrm{x}$ rays, ${ }^{13,14}$ and more recently experimentally implemented in two distinct forms: single energy $x$-ray fluorescence holography (XFH), ${ }^{15}$ and multiple energy $x$-ray holography (MEXH). ${ }^{16,17} \mathrm{MEXH}$ is the time-reversed reciprocal method of $\mathrm{XFH}$, and the relationship between these two methods has been discussed in detail in an earlier study. ${ }^{18} \mathrm{MEXH}$ is found to be more versatile in that aberra- tions and twin-image overlaps can be suppressed by reconstructing images from holograms covering a range of different energies, ${ }^{3,14}$ but it is also more strongly influenced in both positive and negative ways by the polarization of the incident radiation, and this is the topic of the present paper.

While preliminary MEXH images of Fe atoms in hematite $\left(\alpha-\mathrm{Fe}_{2} \mathrm{O}_{3}(001)\right)$ have been reported in a previous study, ${ }^{16}$ we here present additional experimental and theoretical atomic images in order to illustrate the effects of the polarization of the incident radiation used, which can enhance or suppress the images at specific atomic locations. These effects are also theoretically demonstrated through the imaging of simple Fe trimers and a $\mathrm{Ge} \delta$-layer buried in $\mathrm{Si}(001)$, a dilute species-specific structure that is ideally suited for MEXH studies. The limitations and future prospects of this method, specifically as related to polarization effects, are also discussed.

\section{IMAGE RECONSTRUCTION OF MEXH DATA}

As illustrated in Fig. 1(a), raw MEXH $I(\mathbf{k})$ intensities are measured by detecting the net fluorescence from a sample that is illuminated with coherent radiation incident over different directions and energies. The fluorescing emitter detects the overall radiation field strength resulting from a superposition of wave fronts elastically scattered by 
(a)

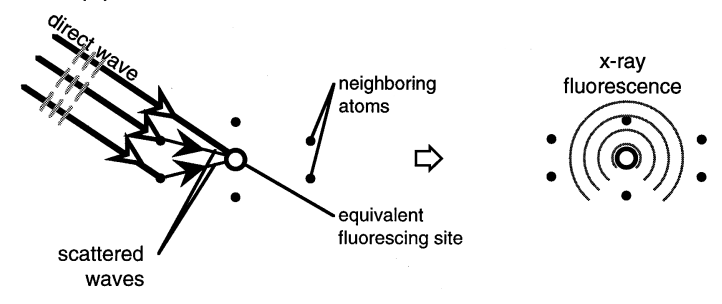

(b)
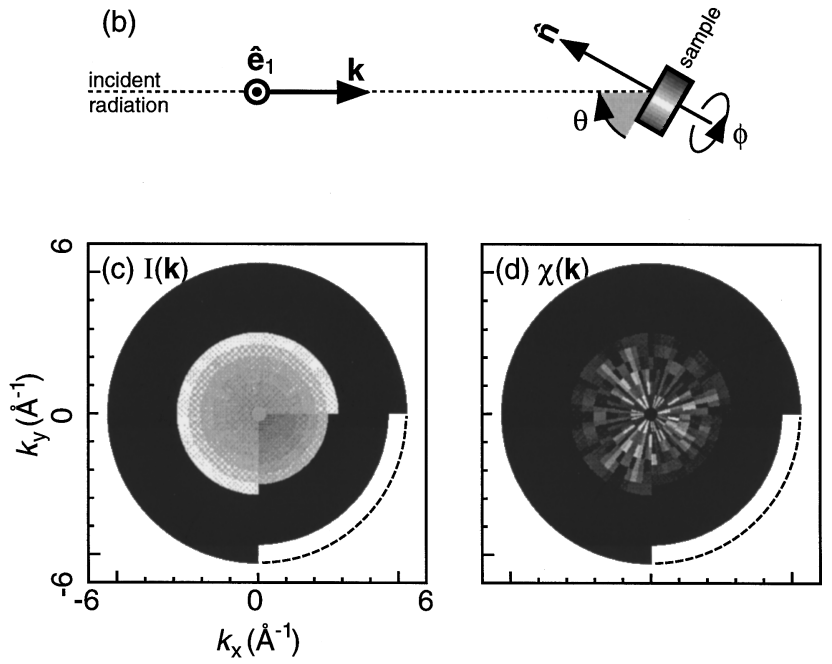

FIG. 1. (a) Multiple energy x ray holography. A coherent farfield excitation x-ray illuminates and photoexcites an emitter, and part of it scatters off of atoms neighboring the emitter in doing so. The emitting atom senses the interference between the direct wave front, and wave fronts elastically scattered by the neighboring atoms. The net photoexcitation is then detected by a stationary, large solid-angle fluorescence detector. Moving the far-field source over a large solid-angle range builds up a holographic interference pattern. (b) Orientation of the sample (where $\hat{\mathbf{n}}$ is the surface normal ([001]) with respect to the horizontal $\left(\hat{\mathbf{e}}_{1}\right)$ polarization vector of the incident radiation $\mathbf{k}$. The polar rotation axis $\hat{\theta}$ is parallel to $\hat{\mathbf{e}}_{1}$. (c) Schematic k-space representation of the raw measured MEXH $I(\mathbf{k})$ intensity data set for $\mathrm{Fe} K \alpha$ fluorescence from $\alpha-\mathrm{Fe}_{2} \mathrm{O}_{3}(001)$ as excited by horizontally polarized radiation at three energies of $k=4.561,4.891,5.220 \AA^{-1}(E=9.00,9.65$, $10.30 \mathrm{keV})$. (d) The normalized MEXH $\chi(\mathbf{k})$ data set.

neighboring atoms and the direct, unscattered wave front (i.e., the direct or reference wave). The amount of fluorescence is thus determined by the interference of the scattered and direct wave fronts at the emitting atomic site. ${ }^{16-18}$ Normalized $\chi(\mathbf{k})$ intensities are obtained via $\chi(\mathbf{k})=[I(\mathbf{k})$ $\left.-I_{0}(\mathbf{k})\right] / \sqrt{I_{0}(\mathbf{k})}$, where $I(\mathbf{k})$ is the raw measured fluorescence, and $I_{0}(\mathbf{k})$ is the fluorescence due specifically to the unscattered, direct wave-front excitation of the emitter. These normalized $\chi(\mathbf{k})$ intensities in $\mathbf{k}$ space can be considered an $\mathbf{r}$-space convolution of the object field $u(\mathbf{r})$ of the scattering atoms surrounding the emitter at the origin, with the convolution kernel describing all of the physics of the $\mathrm{X}$-ray scattering. ${ }^{19}$ This object field can in principle be recovered as an image intensity $U\left(\mathbf{r}^{\prime}\right)$, via a $\mathbf{k}$-space deconvolution of the $\chi(\mathbf{k})$ data set that is in its simplest "optical", form given by

$$
U\left(\mathbf{r}^{\prime}\right) \equiv \iiint_{K} \mathbf{d}^{3} \mathbf{k} \cdot e^{-i\left(\mathbf{k} \cdot \mathbf{r}^{\prime}-k \mathbf{r}^{\prime}\right)} \chi(\mathbf{k}) .
$$

This multiple energy imaging algorithm, and its positive aspects in suppressing twin images and other sources of image aberrations was first pointed out by Barton, ${ }^{3(\mathrm{a}), 3(\mathrm{~b})}$ and subsequently proposed in a slightly different form by Tong, Huang, and Wei. ${ }^{3(\mathrm{c})}$ The kernel $e^{-i\left(\mathbf{k} \cdot \mathbf{r}^{\prime}-k \mathbf{r}^{\prime}\right)}$ implies that the scattered $\mathrm{x}$ rays are isotropic in amplitude, and that the scattering phase shift is zero, or at least constant, both of which conditions are reasonably well met for the scattering of unpolarized $\mathrm{x}$ rays. ${ }^{14}$ However, since MEXH is most easily done on synchrotron-radiation beamlines whose most intense radiation has very strong linear polarization, we explore here the consequences of the highly anisotropic scattering amplitudes which result largely due to Thomson scattering.

\section{THEORETICAL AND EXPERIMENTAL IMAGES OF $\alpha-\mathrm{Fe}_{2} \mathrm{O}_{3}(001)$}

We now show results of applying the reconstruction algorithm of Eq. (1) to experimental and theoretical $\alpha-\mathrm{Fe}_{2} \mathrm{O}_{3}(001)$ (hematite) MEXH $\chi(\mathbf{k})$ data sets, with the radiation polarization being explicitly included in the calculations. The experimental MEXH data was measured by Gog and co-workers at beamline X-14A of the National Synchrotron Light Source at the Brookhaven National Laboratory. ${ }^{16}$ Fe $K \alpha$ fluorescence was collected for horizontally polarized incident-radiation spanning three energies from $k=4.561$ to $5.220 \AA^{-1} \quad(E=9.00-10.30 \mathrm{keV})$ and incident on the $\alpha-\mathrm{Fe}_{2} \mathrm{O}_{3}(001)$ sample over a polar range of $60^{\circ} \leqslant \theta \leqslant 90^{\circ}$. The experimental geometry is illustrated in Fig. 1(b), with the relationship between the horizontal polarization and the rotation axes of the sample being indicated. The polarization here is parallel to the polar rotation axis $(\hat{\theta})$ of the sample, with the azimuthal rotation axis $(\hat{\phi})$ being parallel to the surface normal. These data points were measured at $\delta k$ $=0.329 \AA^{-1} \quad(\delta E=650 \mathrm{eV})$ energy intervals, and at $(\delta \theta, \delta \phi)=\left(5^{\circ}, 5^{\circ}\right)$ angular intervals, making a total of 435 unique measurements in a symmetry-reduced 1/3rd of the total solid angle above the sample. Figure 1(c) shows the raw measured $I(\mathbf{k})$ intensities in $\mathbf{k}$ space, as viewed down along [001]. Data points in the fourth quadrant have been cut away to reveal the $k=4.561 \AA^{-1} I(\mathbf{k})$ intensities. The dark bands at the perimeter indicate the locations in $\mathbf{k}$ space on these isoenergy surfaces where data was not collected. Note that the weak atomic scattering of $\mathrm{x}$ rays renders the anisotropy of the raw $I(\mathbf{k})$ data $\left(\Delta I / I_{0} \approx 0.5 \%\right)$ barely discernible with this gray scale; and thus much more demanding statistical accuracy is required in X-ray holographic measurements than in comparable atomic electron holography measurements (where $\Delta I / I_{0} \approx 30 \%$ ). ${ }^{20}$ However, the more ideal and weaker nature of the atomic scattering of $\mathrm{x}$ rays also produces atomic images that are relatively free of image aberrations, artifacts, and positions shifts compared to atomic electron holography images. ${ }^{14,21-23}$

Due to the limited energy range of this $I(\mathbf{k})$ data set, a separate $I_{0}(\mathbf{k})$ was determined for each of the three different hologram energies via a high-pass filter, ${ }^{24}$ thereby including in $I_{0}(\mathbf{k})$ the reference wave as well as any low-angular- 
frequency effects in the initial excitation of the x-ray and the subsequent absorption as the fluorescence exits the sample, via

$$
I_{0}(\mathbf{k})=\frac{\iint_{\widetilde{\mathbf{k}}} I(\widetilde{\mathbf{k}}) e^{-[(\mathbf{k}-\tilde{\mathbf{k}}) / \sigma]^{2}} \sin \tilde{\theta} d \tilde{\theta} d \tilde{\phi}}{\iint_{\widetilde{\mathbf{k}}} e^{-[(\mathbf{k}-\tilde{\mathbf{k}}) / \sigma]^{2}} \sin \tilde{\theta} d \tilde{\theta} d \widetilde{\phi}} .
$$

All of these effects need to be removed from the raw $I(\mathbf{k})$ intensities before applying the imaging algorithm. Figure 1(d) shows the normalized $\chi(\mathbf{k})$ obtained by this method from the raw $I(k)$ intensities of Fig. 1(c), with holographic modulations more visible.

For comparison, a single-scattering model ${ }^{14,25}$ was used to calculate a theoretical MEXH $\chi(\mathbf{k})$ data set from an ideal $\alpha$ $-\mathrm{Fe}_{2} \mathrm{O}_{3}(001)$ cluster containing only $384 \mathrm{Fe}$ atoms occupying the two inequivalent $\mathrm{Fe}$ emitter sites appropriate to this crystal structure. The $\mathrm{O}$ atoms were omitted, as they will be negligibly strong in the resulting reconstructed images due to their relatively smaller scattering power. ${ }^{16}$ The incident radiation in this model calculation is polarized horizontally with respect to the $\hat{\theta}$ and $\hat{\phi}$ rotations performed on this cluster, just as for the measurement of the experimental $I(\mathbf{k})$ data set discussed above [cf. Fig. 1(b)]. As the sample was rotated over the $60^{\circ} \leqslant \theta \leqslant 90^{\circ}$ range of the experimental data, the polarization (and the resultant Thomson cross section) rotated also with respect to the crystal structure, but with an average overall effect still of having much stronger scattering for atoms above and below a given emitter along the surface normal or [001] direction.

Figure 2 schematically shows the $\mathbf{r}$-space regions in the vertical (120) and horizontal $(00 \overline{2})$ planes that would have enhanced/suppressed image intensities due to the effect of using horizontally polarized incident radiation, relative to the image intensities obtained from an unpolarized incident radiation MEXH data set. The image intensity in Fig. 2 is thus the ratio $U_{\text {horizontal }}\left(\mathbf{r}^{\prime}\right) / U_{\text {unpolarized }}\left(\mathbf{r}^{\prime}\right)$, with white representing a value of unity, and gray, values of $<1$. As can be seen in Fig. 2, the use of horizontally polarized incident radiation would most strongly suppress atomic images in the horizontal (001) plane containing the emitter, with this suppression being less in horizontal planes farther above and below the emitter plane. Images along the [001] axis would be least suppressed by using horizontally polarized incident radiation. Thus, using horizontally polarized radiation in the geometry of Fig. 1(b) would in fact be disadvantageous in the study of horizontal planar structures such as those prevalent in $\alpha-\mathrm{Fe}_{2} \mathrm{O}_{3}(001)$, which is comprised of closely stacked horizontal Fe bilayers.

We now show the reconstructed atomic images obtained from applying Eq. (1) to the experimental (Fig. 3) and theoretical (Fig. 4) single-scattering model MEXH $\chi(\mathbf{k})$ data sets for $\alpha-\mathrm{Fe}_{2} \mathrm{O}_{3}(001)$ in (a) the vertical (120) plane, and (b) the horizontal $(00 \overline{2})$ plane that is $6.89 \AA$ below the emitter. These images are the all superpositions of images for both of the inequivalent $\mathrm{Fe}$ emitter sites, with these emitter sites indicated by a common dashed square at the origin, and the relative positions of the Fe scatterers indicated by circles. Fe scatterers just above or below the $(00 \overline{2})$ Fe bilayer are indi-

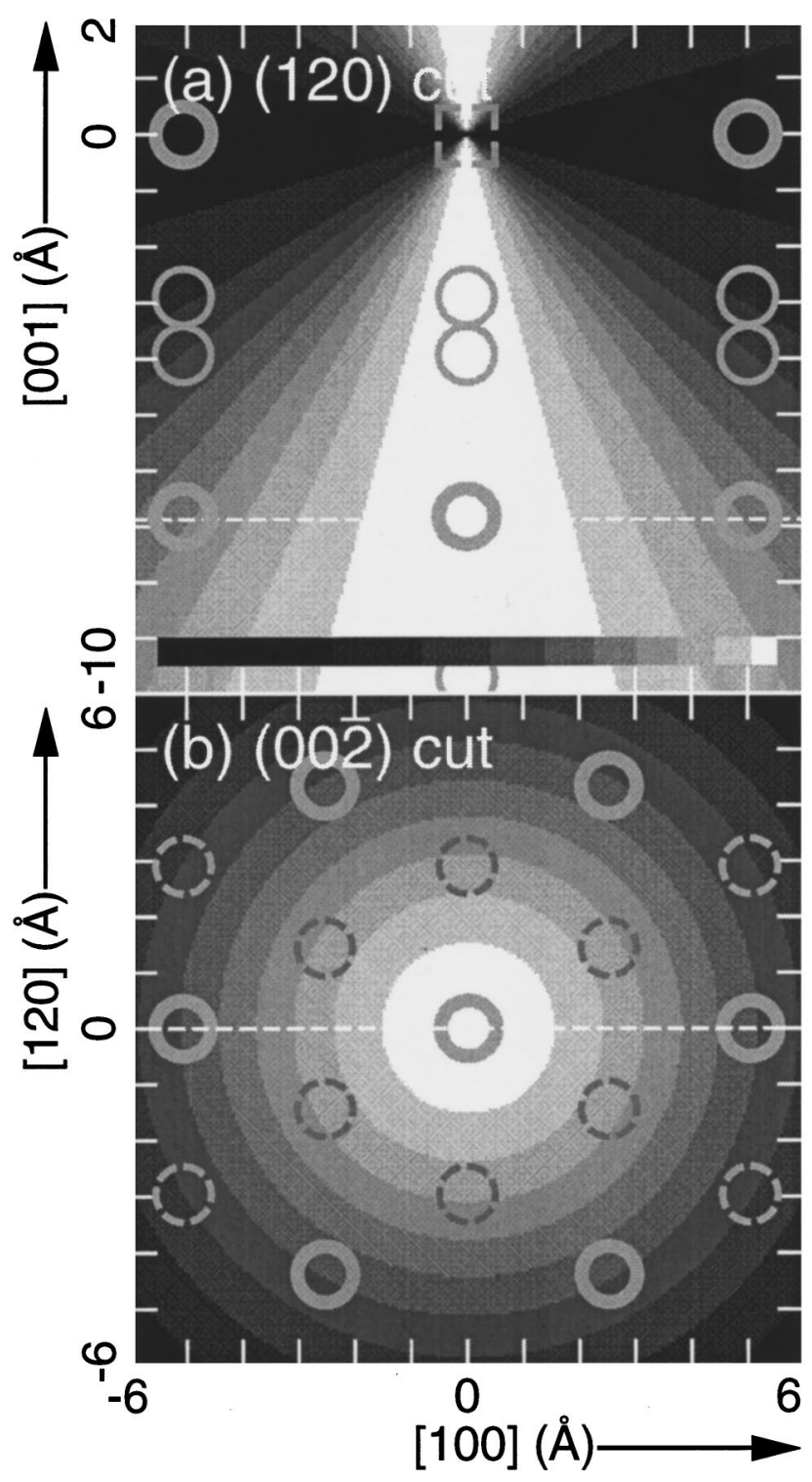

FIG. 2. Theoretically calculated ratio of image intensities obtained using horizontally polarized incident radiation and image intensities obtained using unpolarized incident radiation on $\alpha-\mathrm{Fe}_{2} \mathrm{O}_{3}(001)$, in (a) the vertical (120) plane, and (b) the horizontal $(00 \overline{2})$ plane of $6.89 \AA$ below the emitter. The superposition of both inequivalent $\mathrm{Fe}$ emitter sites is indicated by a dashed square, and $\mathrm{Fe}$ scatterers are indicated by circles. Fe scatterers in the bilayer just above or below this plane are indicated by dashed circles, and Fe scatterers in relative positions common to both inequivalent $\mathrm{Fe}$ emitters are indicated by bold circles. Axes are marked off in $1-\AA$ units.

cated by dashed circles, and $\mathrm{Fe}$ scatterers in relative positions common to both inequivalent $\mathrm{Fe}$ emitters are indicated by bold circles. The atomic image resolutions expected from $\chi(\mathbf{k})$ data sets of this energy and angular range ${ }^{24}$ are $\delta x$ $=\delta y \approx 0.6 \AA$ in the horizontal ([001] and [120]) directions, and $\delta z \approx 2.5 \AA$ in the vertical ([001]) direction, and these resolutions are indicated by white error bars in the figures. The experimental and theoretical images are in excellent agreement. In particular, both experiment and theory show that three of the $\mathrm{Fe}$ atoms from the neighboring upper bilayer intrude into the $(00 \overline{2})$ image plane. This is due to the limited 


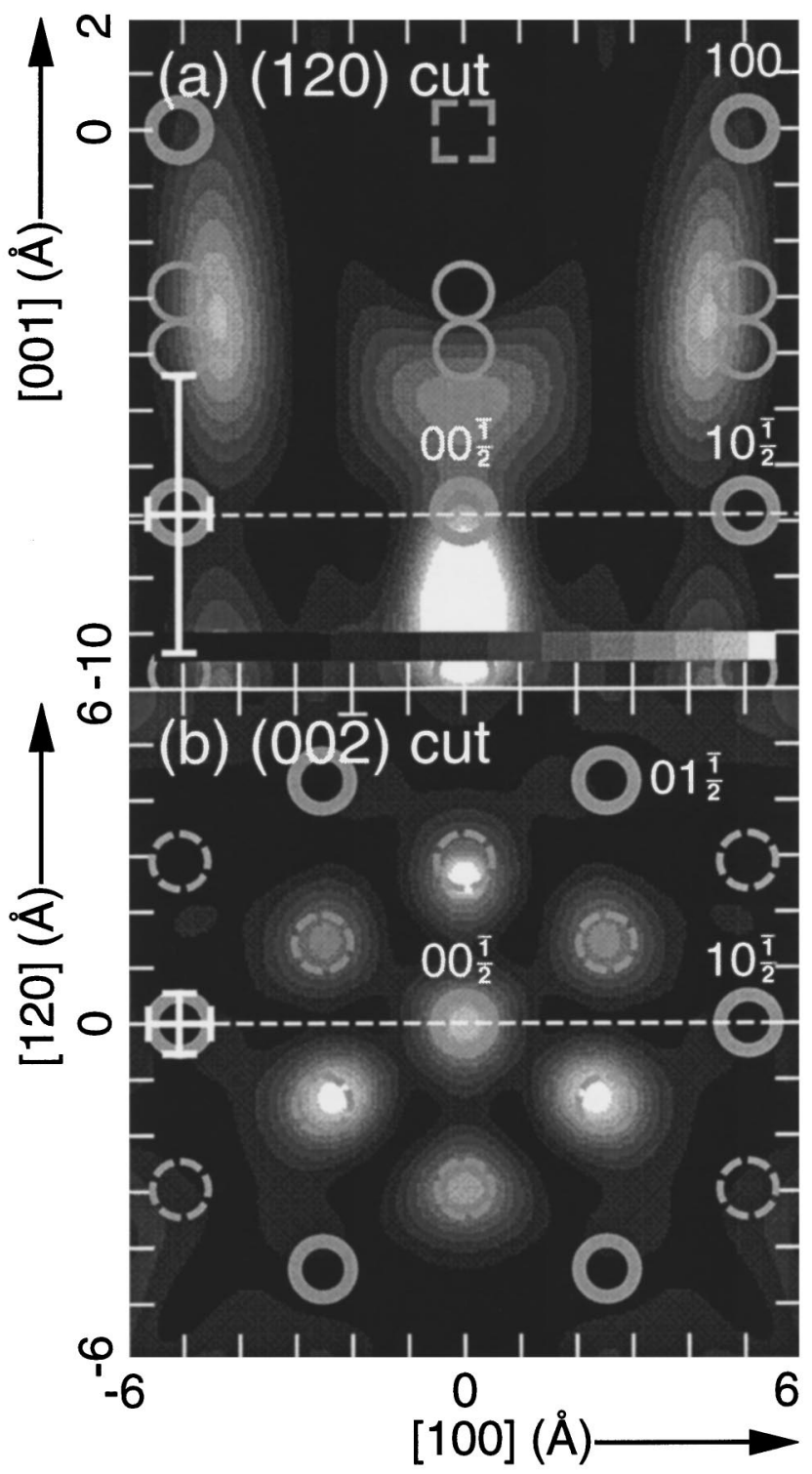

FIG. 3. Reconstructed $\alpha-\mathrm{Fe}_{2} \mathrm{O}_{3}(001)$ atomic images obtained from an experimental MEXH data set measured with horizontally polarized incident radiation, in (a) the vertical (120) plane, and (b) the horizontal $(00 \overline{2})$ plane. The image resolution expected from the energy and angular ranges of this $\chi(\mathbf{k})$ data set are indicated by the white error bars.

energy and angular range of the $\chi(\mathbf{k})$ data set in $\mathbf{k}$ space, which results in atomic images much less resolved in the vertical [001] direction. ${ }^{24}$ Also, as expected from the suppression of horizontal image intensities that arises from the use of horizontally polarized incident radiation, the $\mathrm{Fe}$ atomic images in the (001) plane are not visible in Figs. 3 and $4(\mathrm{a})$, and the $(00 \overline{2})$ in-plane atoms $\left(10 \frac{\overline{1}}{2}, 01 \frac{\overline{1}}{2}\right.$, etc. $)$ are also not visible in Figs. 3 and 4(b), due to their horizontal distance from the [001] axis. The only Fe atomic images that have not been appreciably suppressed lie more nearly along the vertical [001] direction above and below the emitter [cf. Figs. 2(a), 3(a), 4(a)]. In spite of the image suppression due to the polarization of the incident radiation, and the resolution loss due to the limited energy and angular range of these $\chi(\mathbf{k})$ data sets, the resulting images are found to exhibit a

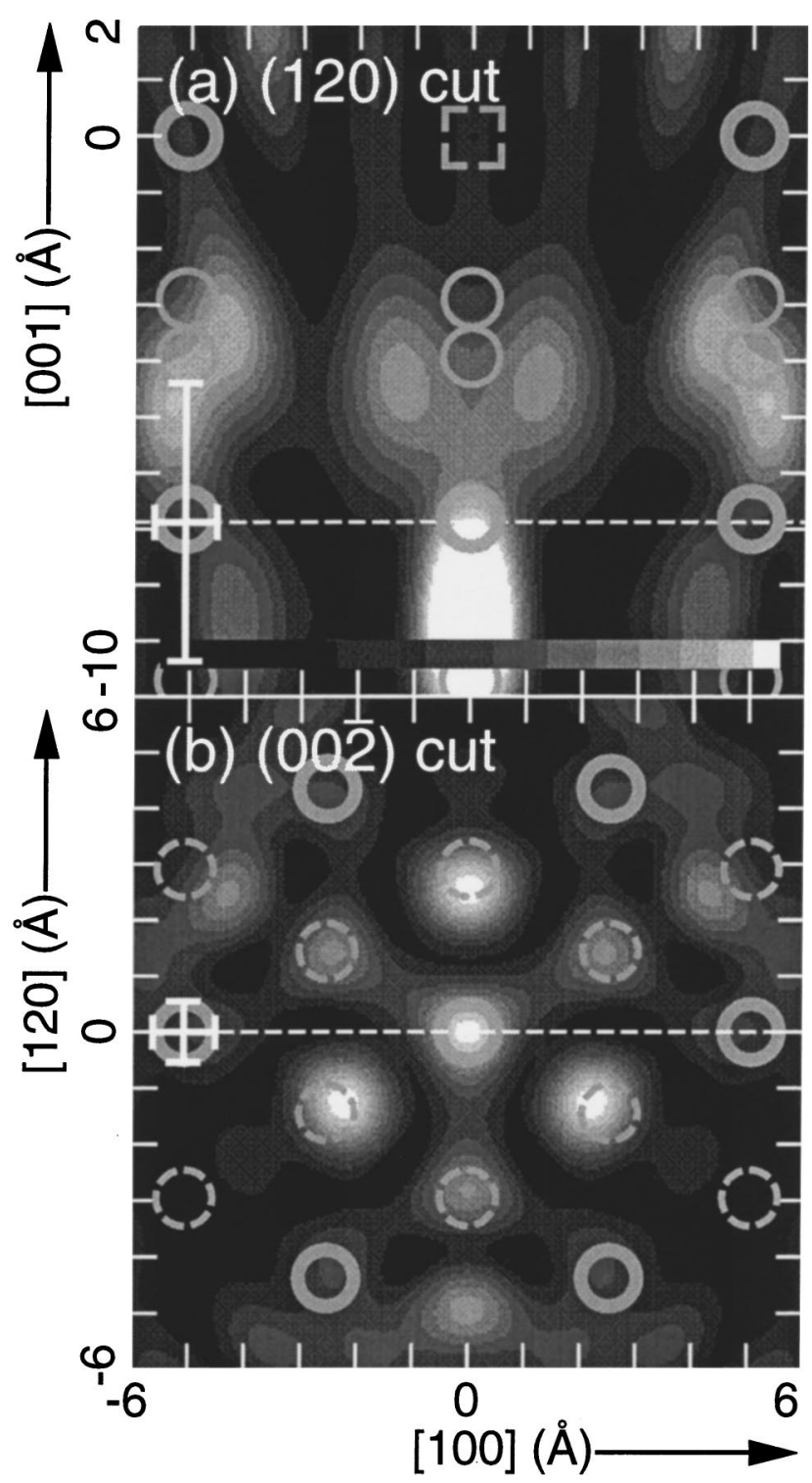

FIG. 4. As in Fig. 3, but for images obtained from a theoretical horizontally polarized MEXH data set.

suppression of real-twin image overlaps, as they are reconstructed from a multiple energy $\chi(\mathbf{k})$ data set. ${ }^{3,14,16-18}$

\section{EFFECT OF INCIDENT POLARIZATION ON MEXH HOLOGRAPHIC INTENSITIES}

In order to best image horizontal and vertical atomic positions simultaneously, utilizing unpolarized incident radiation would be ideal. However, because synchrotron radiation is the most practical experimental MEXH incident-radiation source due to its energy tunability, high brightness, and potentially high-energy resolution (e.g., via undulators and/or crystal monochromators), linearly polarized incident radiation must be considered. We thus now discuss in more detail the effect of incident radiation polarization on the creation of MEXH $\chi(\mathbf{k})$ intensities for two model systems.

Figure 5 shows the $\mathrm{Fe}$ atomic scattering factor magnitudes $\left|f_{\mathrm{Fe}}(\Theta)\right|$ for $\mathrm{x}$ rays at $k=5.220 \AA^{-1}(E=10.30 \mathrm{keV})$, where $\Theta$ is the angle between the incident $(\mathbf{k})$ and scattered 

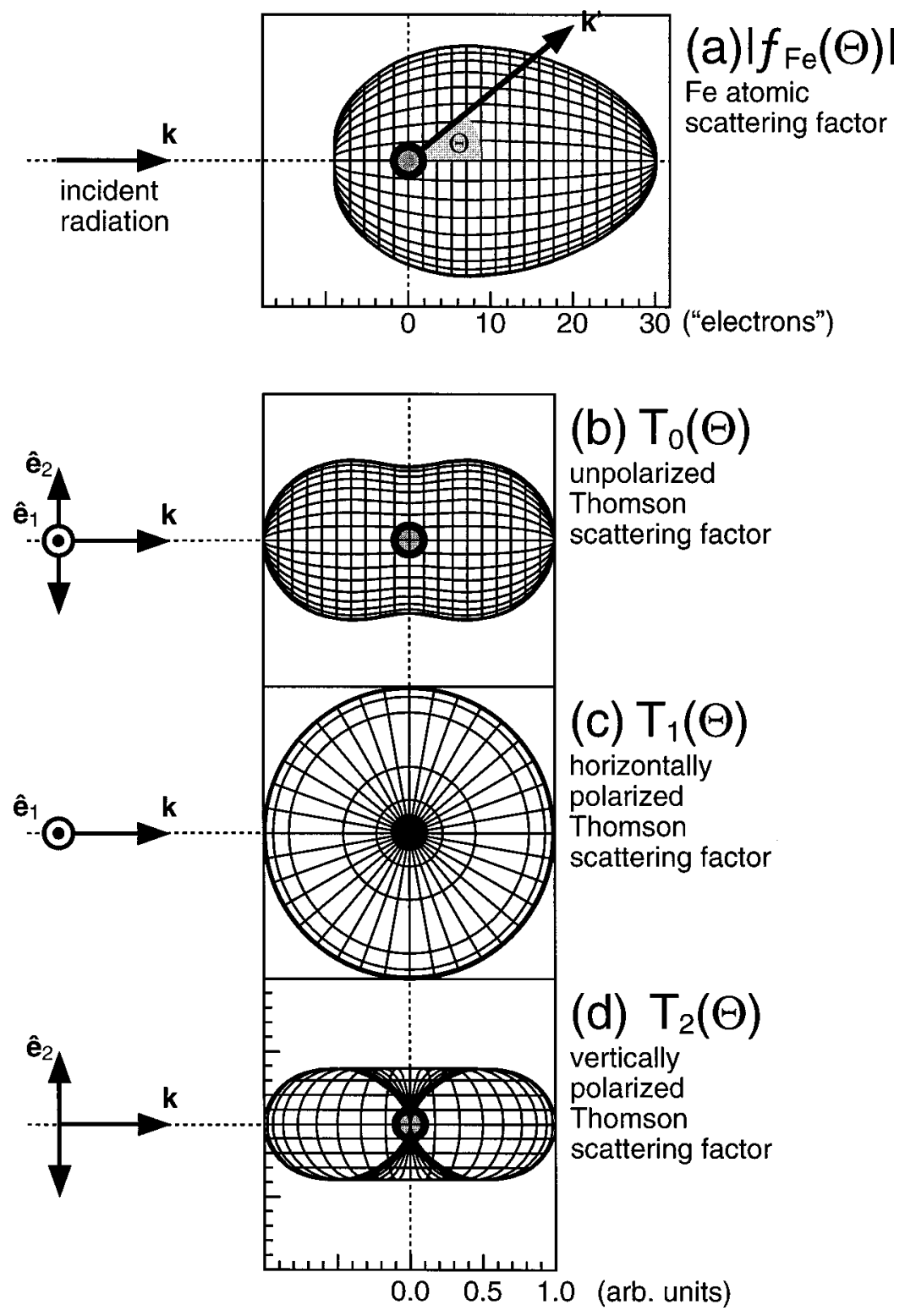

FIG. 5. Scattering factor magnitudes for $k$ $=5.220 \AA^{-1}(E=10.30 \mathrm{keV}) \mathrm{x}$-rays incident on atomic Fe. $\Theta$ is the angle between the incident (k) and scattered $\left(\mathbf{k}^{\prime}\right)$ wave vectors. (a) Fe atomic scattering factor, in relative units of electrons. (b) - (d) Thomson electron scattering factors, in absolute units of $r_{e} \equiv 2.818 \times 10^{-5} \AA$, for (b) unpolarized, (c) vertically polarized, and (d) horizontally polarized incident $\mathrm{x}$ rays.

$\left(\mathbf{k}^{\prime}\right)$ wave fronts. $^{25}$ The raw Fe scattering factor magnitude, as given in relative electron-scattering units [Fig. 5(a)], is converted to absolute units by multiplying by the Thomson scattering factor for a single electron interacting with either unpolarized or linearly polarized light, as the case may be. The general form of the well-known Thomson scattering factor is $\sin \Theta_{\hat{\mathbf{e}}}^{\mathbf{k}^{\prime}}$, where $\Theta_{\hat{\mathbf{e}}}^{\mathbf{k}^{\prime}}$ is the angle between the polarization vector of the incident radiation $\hat{\mathbf{e}}$, and the direction $\mathbf{k}^{\prime}$ of the scattered radiation. Figures 5(b)-5(d) show the Thomson scattering factors for unpolarized, horizontally polarized, and vertically polarized light, respectively. The "peanut' -shaped unpolarized electron-scattering factor [Fig. 5(b)] has an azimuthal symmetry about the incident wave-vector axis, while this symmetry is broken for the "bagel"'-shaped linearly polarized scattering factors [Figs. 5(c)-(d)], which for horizontal and vertical polarization show preferential scattering in the vertical and horizontal directions, respectively.

The effect of polarized incident radiation in MEXH can be demonstrated by considering the ideal $\chi(\mathbf{k})$ holograms created by simple [001] and [100] linear Fe trimers oriented along the vertical or horizontal direction, respectively, as shown to the left of Figs. 6-8. In these calculations, the trimers were rotated with respect to the incident-radiation polarization so as to simulate a real experimental situation with a movable sample and a fixed beamline. Figures 6-8 show the expected holographic intensities in the full upper $2 \pi$ hemisphere above these Fe trimers, as viewed down along the [001] directions, for a hologram energy of $k$ $=5.220 \AA^{-1}(E=10.30 \mathrm{keV})$. The holographic interference fringes in the case of unpolarized incident radiation are visible as azimuthal bands for the vertical [001] Fe trimer in Fig. 6(a), and as vertical bands centered along the [100] direction for the horizontal [100] Fe trimer in Fig. 6(b). Figures 9-11 show the atomic image intensities reconstructed via Eq. (1) along these trimers. The full width at half maximum of these image peaks are close to the resolutions expected from the energy and angular range of these $\chi(\mathbf{k})$ data sets $(d x \approx 0.31 \AA ; d z \approx 0.61 \AA) .{ }^{24}$

Figure 7 shows the MEXH $\chi(\mathbf{k})$ holograms from these same Fe trimers, but in the case of incident-radiation polarized horizontally with respect to a stationary synchrotron source, where the polarization vector $\hat{\mathbf{e}}$ is parallel to the $\hat{\theta}$ 

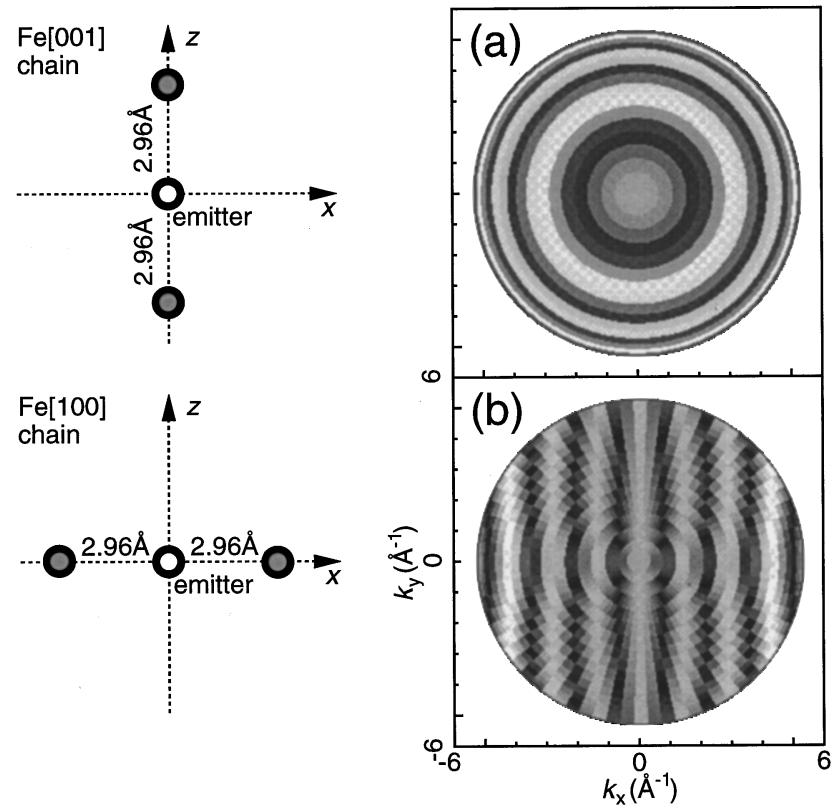

FIG. 6. Normalized holographic intensities $\chi(\mathbf{k})$ as calculated for $\mathrm{Fe} K \alpha$ fluorescence excited by $k=5.220 \AA^{-1} \quad(E$ $=10.30 \mathrm{keV}$ ) unpolarized $\mathrm{x}$ rays incident on two different Fe trimers, viewed down along [001] . (a) [001] Fe trimer $\chi(\mathbf{k})$. (b) $[100] \mathrm{Fe}$ trimer $\chi(\mathbf{k})$.

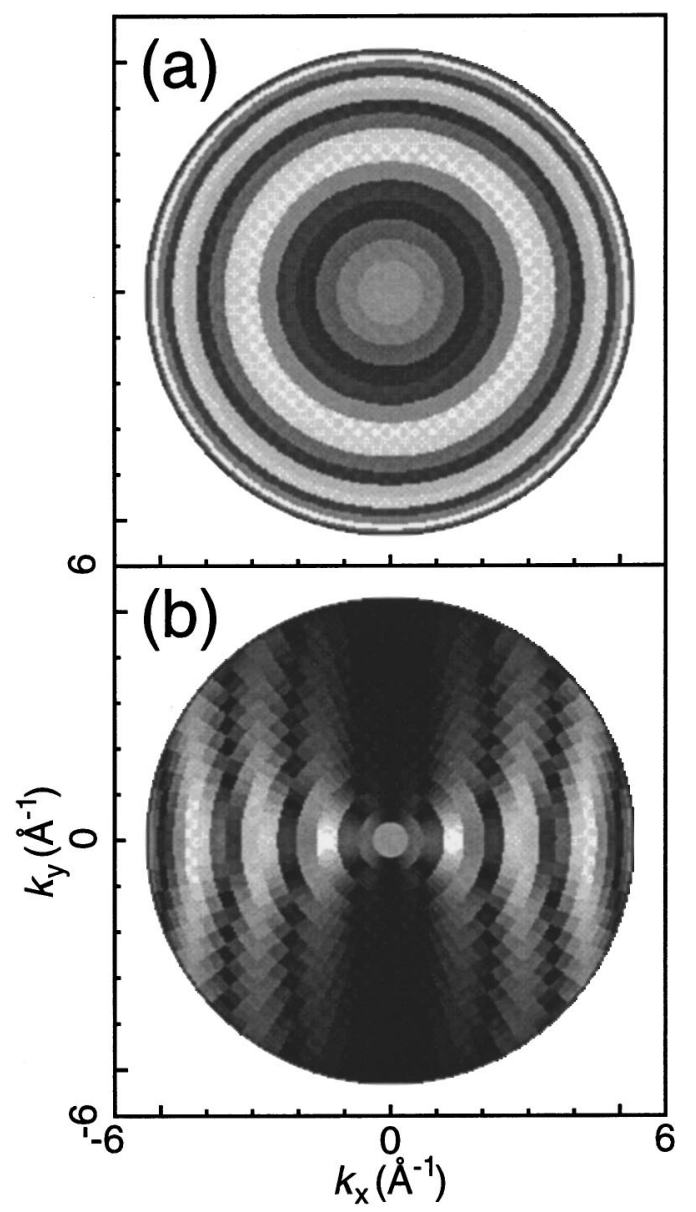

FIG. 7. As in Fig. 6, but for the case of horizontally polarized incident radiation.

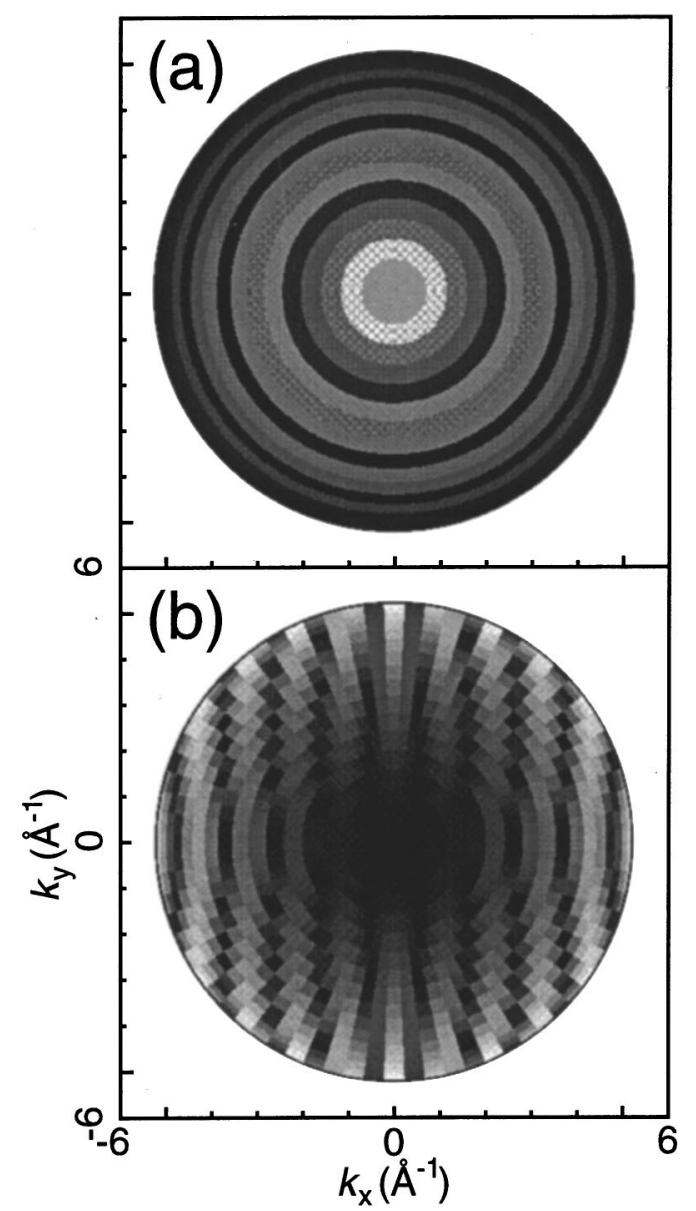

FIG. 8. As in Fig. 6, but for the case of vertically polarized incident radiation.

rotation axis, as shown in Fig. 1(b). Due to the azimuthal symmetry of the horizontal polarization with respect to the [001] Fe trimer for all incident-radiation angles, the resulting hologram in Fig. 7(a) is virtually identical (although relatively less intense, due to the loss of one polarization mode) to the unpolarized incident-radiation hologram in Fig. 6(a). However, the $\chi(\mathbf{k})$ hologram intensities for the [100] Fe trimer in the case of horizontally polarized light are suppressed in directions perpendicular to the [100] trimer axis [Fig. 7(b)]. There, the Thomson scattering factor selects against scattering between the trimer atoms, such that there is much reduced holographic information in these regions. This effect is seen in the reconstructed images of Fig. 10, where the [001] Fe trimer image peaks [Fig. 10(a)] are now approximately four times higher in intensity than the [100] Fe trimer image peaks [Fig. 10(b)]. Thus horizontal polarization emphasizes atomic images along the vertical azimuthal axis, while suppressing horizontal planar atomic images, as noted before for the more complex $\alpha-\mathrm{Fe}_{2} \mathrm{O}_{3}(001)$ case.

Figure 8 shows the MEXH $\chi(\mathbf{k})$ holograms from the [001] and [100] Fe trimers, in the case of incident radiation polarized vertically with respect to a stationary synchrotron source, where the polarization vector $\hat{\mathbf{e}}_{2}$ is now always perpendicular with the $\hat{\theta}$ rotation axis. Again, due to the azimuthal symmetry of the vertical polarization with respect to the [001] Fe trimer for all incident radiation angles, the resulting hologram in Fig. 9(a) is nearly identical in the upper 

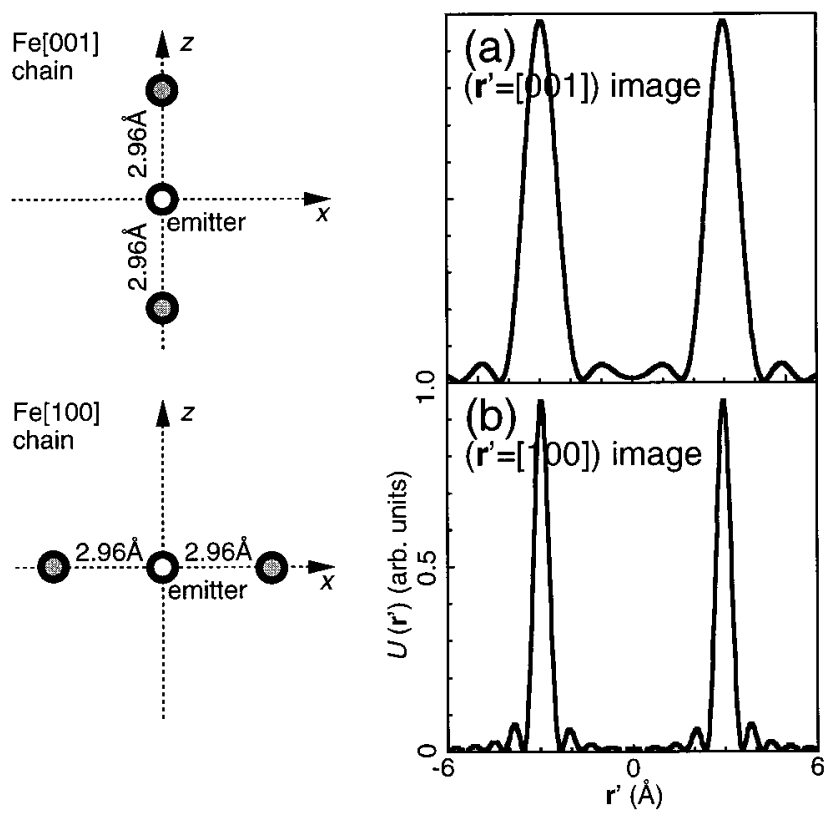

FIG. 9. Reconstructed atomic images obtained from the theoretical unpolarized incident-radiation MEXH $\chi(\mathbf{k})$ data sets of Fig. 6, for (a) the [001] Fe trimer, and (b) the [100] Fe trimer. Image intensities are scaled in arbitrary units relative to each other. The distances $\mathbf{r}^{\prime}$ along the trimer axes (whether [001] or [100]) are marked off in $1-\AA$ units.

polar regions to the unpolarized incident radiation hologram in Fig. 7(a), but is weaker in intensity for lower polar angles, where the polarized Thomson scattering factor selects against scattering between the trimer atoms, leading to reduced holographic information in these regions. For the [100] Fe trimer hologram, the polarized Thomson scattering factor suppresses holographic intensities in the upper polar regions parallel to the [100] trimer orientation. This effect is seen in the reconstructed images of Fig. 11, where the [001] Fe trimer image peaks [Fig. 11(a)] are now approximately half the intensity of the [100] Fe trimer image peaks [Fig. 11(b)]. Thus for horizontal polarized incident radiation, holographic intensities in specific regions are suppressed for both the [001] and [100] trimers, but the net effect for images reconstructed from the full upper $2 \pi$ hemisphere is to suppress peak intensities along the vertical [001] axis more than in the horizontal plane.

Therefore compared to unpolarized incident radiation, linearly polarized incident radiation reduces the amount of spatial information corresponding to specific atomic sites available in MEXH $\chi(\mathbf{k})$ intensities. Horizontally polarized incident radiation suppresses holographic and reconstructed image intensities for atoms in the horizontal (001) plane of the emitter, while emphasizing intensities for atoms in the vertical [001] axis through the emitter. For low polar angles, vertically polarized incident radiation suppresses low takeoff angle $\chi(\mathbf{k})$ intensities from atoms in the vertical [001] axis through the emitter, while emphasizing $\chi(\mathbf{k})$ intensities from atoms in the horizontal plane of the emitter; these effects are reversed when higher polar angles are considered for vertically polarized incident radiation. When taken over the entire full upper $2 \pi$ hemisphere of $\chi(\mathbf{k})$ intensities, reconstructed image intensities for atoms along the vertical [001] axis are

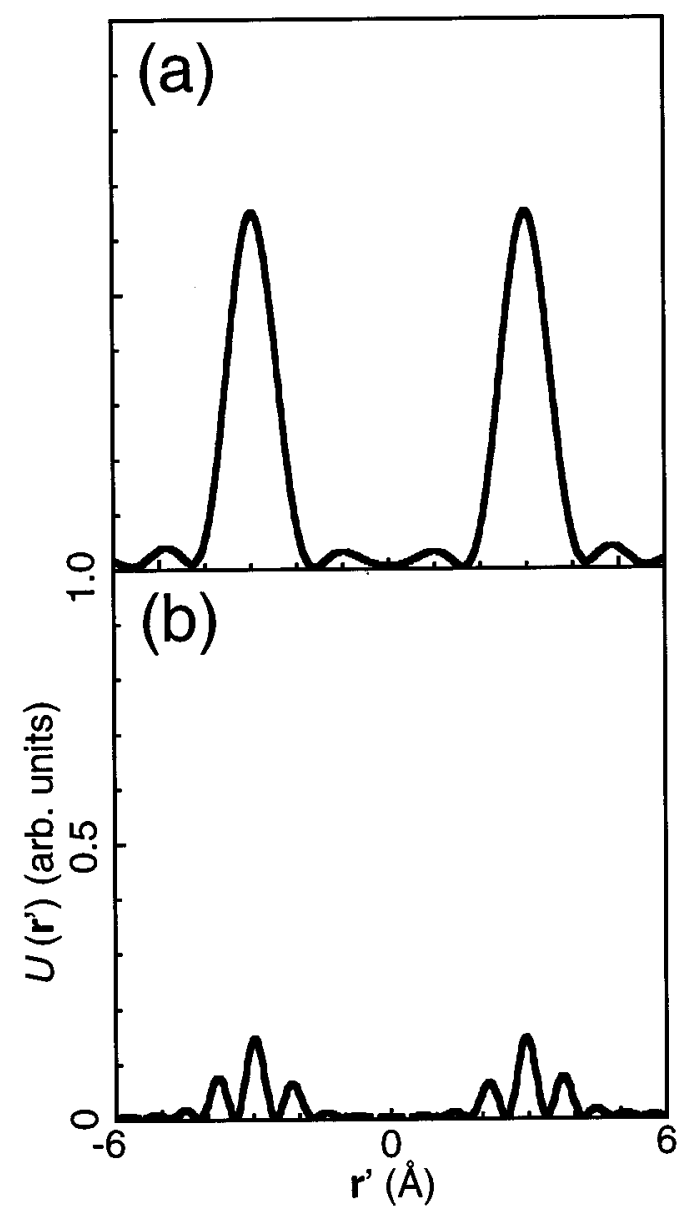

FIG. 10. As in Fig. 9, but for images reconstructed from the horizontally polarized incident-radiation MEXH $\chi(\mathbf{k})$ data of Fig. 7.

suppressed more, relative to atoms in the horizontal plane of the emitter.

\section{EFFECT OF INCIDENT POLARIZATION ON MEXH ATOMIC IMAGES}

In order to illustrate the possible utility of linearly polarized incident radiation in obtaining MEXH atomic images, we consider a general class of structures where it should be advantageous to utilize horizontally polarized incident radiation, and for which vertical structural information can be much more desirable than horizontal structural information: surface atomic layers and buried epitaxial atomic layers. A specific case theoretically illustrated here is a Ge $\delta$-layer buried in $\mathrm{Si}(001)$. The Ge atoms in the $\delta$ layer are here assumed to lie in horizontal epitaxial sites with respect to the surrounding $\mathrm{Si}(001)$, such that structural information in the horizontal plane of a Ge emitter is relatively unimportant compared to the strained vertical distances between the $\mathrm{Ge}$ $\delta$-layer atoms and their $\mathrm{Si}$ neighbors above and below them. ${ }^{26}$ Thus using horizontally polarized incident radiation to record a MEXH Ge $K \alpha$ data set for this system or others like it may prove to be advantageous.

MEXH $\chi(\mathbf{k})$ intensities were calculated for unpolarized, horizontally polarized, and vertically polarized radiation incident on an ideal $\mathrm{Ge} \delta$-layer buried in a $\mathrm{Si}(001)$ cluster containing a total of 96 atoms, where for simplicity no ver- 


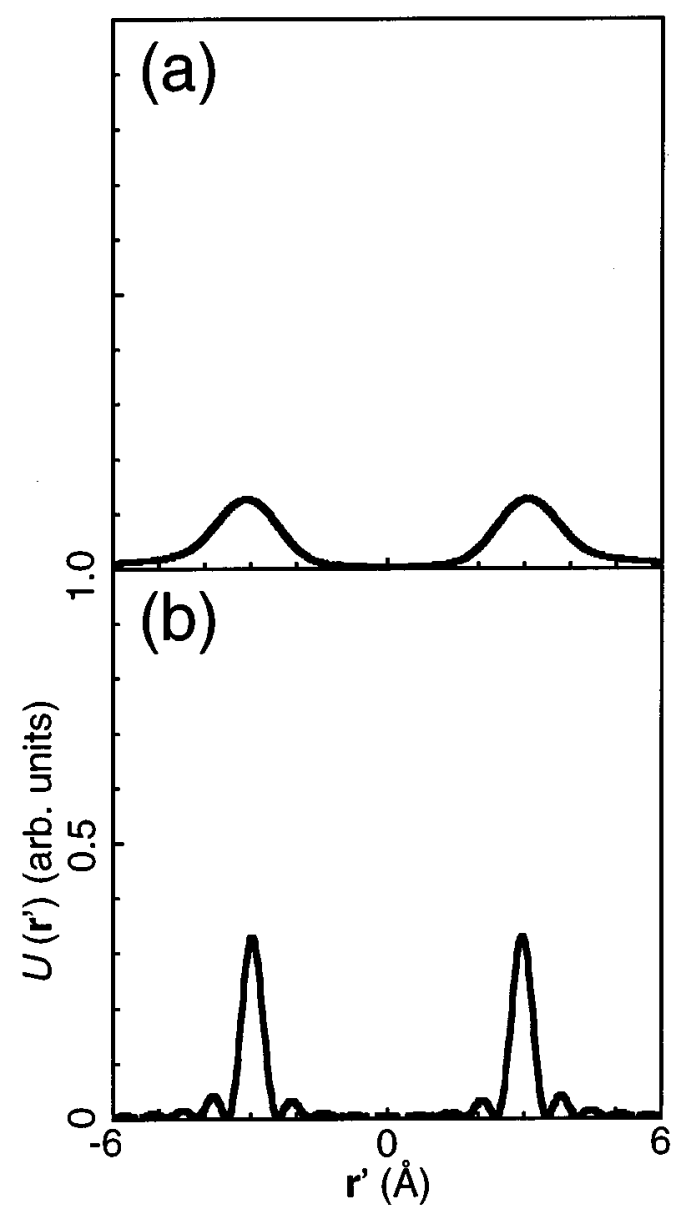

FIG. 11. As Fig. 9, but for images reconstructed from the horizontally polarized incident-radiation MEXH $\chi(\mathbf{k})$ data of Fig. 8.

tical interlayer strain was considered. The incident radiation ranged in energy from $k=6.081$ to $9.122 \AA^{-1}(E$ $=12.00-18.00 \mathrm{keV}$ ), which is above the Ge $K$ absorption edge $\left(k=5.625 \AA^{-1}\right.$ or $\left.E=11.00 \mathrm{keV}\right)$, and enables the imaging of the equivalent atomic environment surrounding each $\mathrm{Ge}$ emitter. This radiation is incident over a polar range of $10^{\circ} \leqslant \theta \leqslant 90^{\circ}$, and these holograms were calculated at seven energies corresponding to $\delta k=0.507 \AA^{-1} \quad(\delta E$ $=1.000 \mathrm{keV})$ and $(\delta \theta, \delta \phi)=\left(5^{\circ}, 5^{\circ}\right)$ intervals, for a total of 1897 unique data points in a symmetry-reduced $\frac{1}{4}$ th of the total solid-angle above the cluster. The higher energy and larger energy and angular ranges of this MEXH data ensure better resolved atomic images $(\delta x=\delta y \approx 0.2 \AA$; $\delta z \approx 0.4 \AA$ ) than those of Figs. 3 and $4 .^{24}$

Figure 12 shows the reconstructed atomic image in the vertical (110) plane obtained by applying Eq. (1) to the theoretical unpolarized incident-radiation MEXH $\chi(\mathbf{k})$ data set. The typical Ge emitter site is indicated by a dashed square, Ge scatterers are indicated by squares, and the bulk Si scatterers are indicated by circles. In this image the Ge $\delta$-layer atoms are well defined, and the $\mathrm{Si}$ atoms in the layer immediately above the $\delta$ layer are moderately resolved. Images of the Si layers further above, and below the $\delta$ layer are faintly discernible with this gray scale.

In contrast, atomic images reconstructed from linearly polarized incident-radiation MEXH $\chi(\mathbf{k})$ data sets are enhanced/suppressed in specific regions, relative to the un-

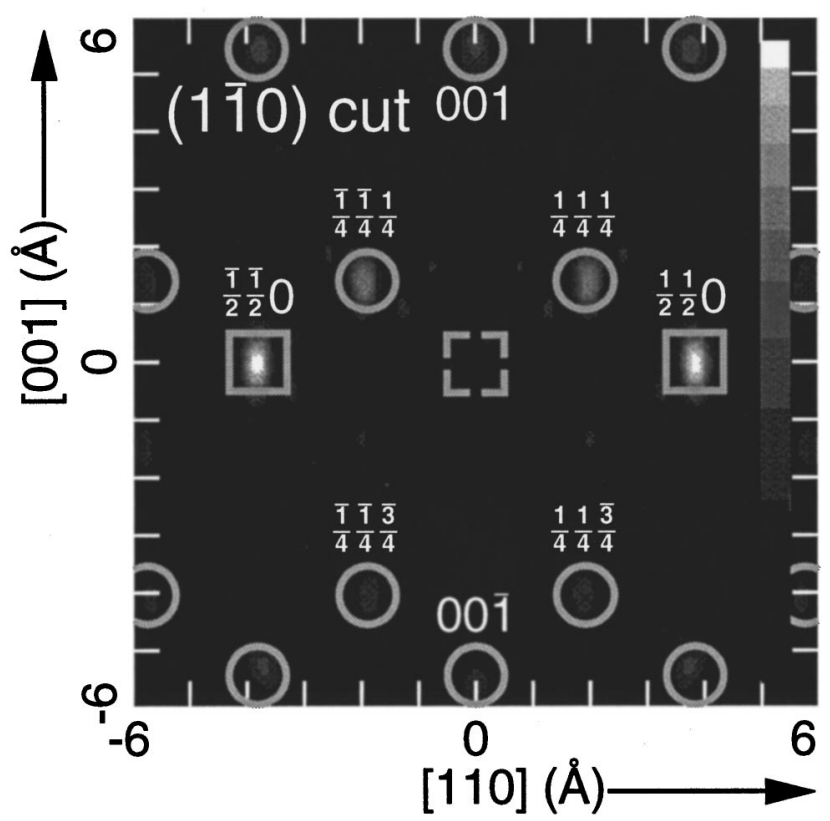

FIG. 12. Reconstructed $\mathrm{Si}(001) / \mathrm{Ge}-\delta / \mathrm{Si}(001)$ atomic images obtained from a theoretical unpolarized incident-radiation MEXH $\chi(\mathbf{k})$ data set, in the vertical (110) plane. The typical equivalent $\mathrm{Ge}$ emitter site is indicated by a dashed square, Ge scatterers are indicated by squares, and Si scatterers are indicated by circles. Axes are marked off in $1-\AA$ units.

polarized incident-radiation atomic images of Fig. 12. Figures 13(a) and 14(a) show the relative image enhancement ratios $U_{\text {horizontal }}\left(\mathbf{r}^{\prime}\right) / U_{\text {unpolarized }}\left(\mathbf{r}^{\prime}\right)$ and $U_{\text {vertical }}\left(\mathbf{r}^{\prime}\right) /$ $U_{\text {unpolarized }}\left(\mathbf{r}^{\prime}\right)$, respectively, for atomic images reconstructed in the (110) plane from horizontally and vertically polarized incident-radiation $\chi(\mathbf{k})$ data sets. As seen earlier in Fig. 2(a), Fig. 13(a) shows that atomic images along the vertical [001] axis through the emitter (i.e., the 001 and $00 \overline{1}$ atoms) will be most enhanced relative to images near the horizontal (001) plane (the $\frac{\overline{1}}{2} \frac{1}{2} 0, \frac{1}{2} \frac{1}{2} 0, \frac{1}{4} \frac{1}{4} \frac{1}{4}$, and $\begin{array}{llll}1 & 1 & 1 \\ 4 & 4 & 4\end{array}$ atoms) when reconstructed from MEXH data obtained with horizontally polarized incident radiation. The image regions specifically enhanced in Fig. 13(a) differ slightly from that of Fig. 2(a), due to the different energy and angular ranges of their respective $\chi(\mathbf{k})$ data sets. Figure 13(b) shows the reconstructed atomic images obtained from horizontally polarized MEXH data in the vertical $(\overline{1} 10)$ plane, where as expected the $\mathrm{Ge} \delta$-layer atomic images are suppressed relative to the prominent $\mathrm{Si}$ atomic images along the [001] axis. Thus strain in the vertical interlayer distances in this system would be most easily determined in an MEXH experiment using horizontally polarized incident radiation.

Figure 14(a) shows that atomic images in the horizontal (001) plane (i.e., the $\frac{1}{2} \frac{1}{2} 0$ and $\frac{1}{2} \frac{1}{2} 0$ atoms) will be enhanced relative to images in the vertical [001] axis (the 001 and $00 \overline{1}$ atoms) when reconstructed from MEXH data obtained with vertically polarized incident radiation. Figure 14(b) shows the reconstructed atomic images in the vertical (110) plane obtained from such a data set. Similar to the unpolarized images of Fig. 12, the most prominent features in Fig. 14(b) are the $\mathrm{Ge} \delta$-layer atoms, followed by the $\mathrm{Si}$ atoms in the layer immediately above them. However, the faint $\mathrm{Si}$ atomic 


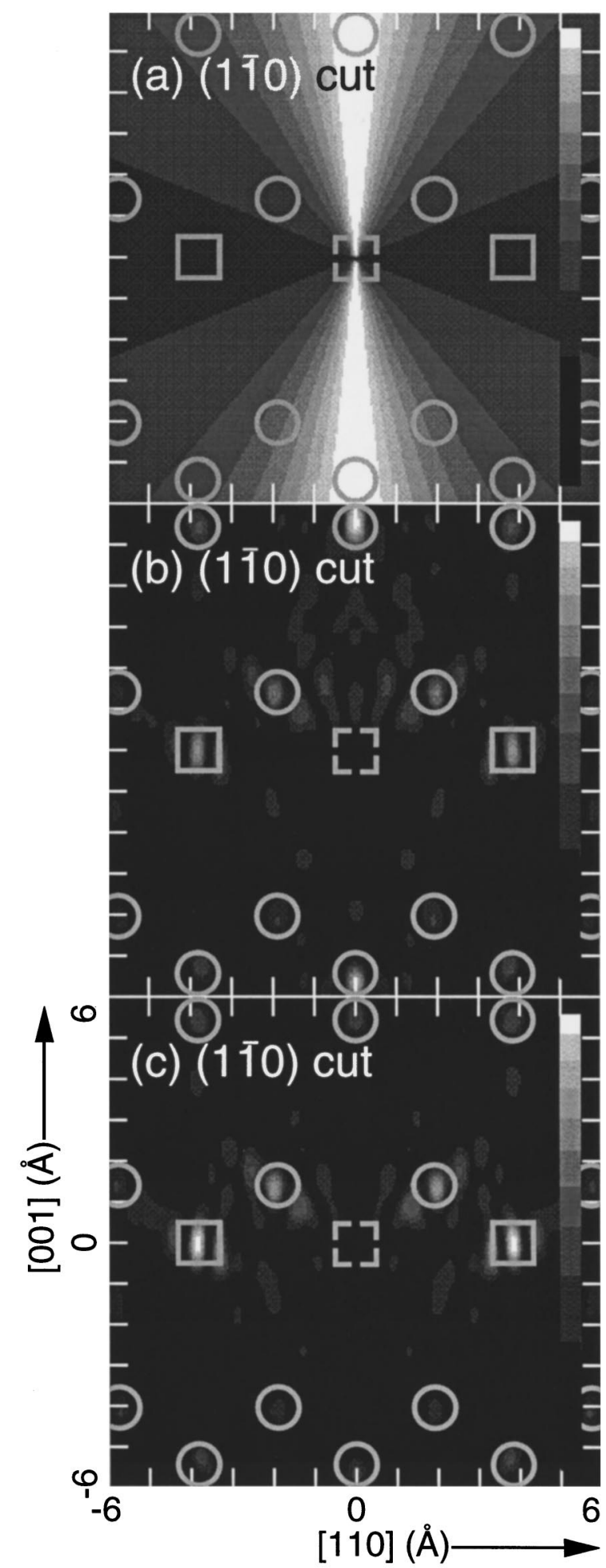

FIG. 13. (a) Ratio of image intensities obtained using horizontally polarized incident radiation to image intensities obtained using unpolarized incident radiation, in the vertical (110) plane. (b) Reconstructed $\mathrm{Si}(001) / \mathrm{Ge}-\delta / \mathrm{Si}(001)$ atomic images obtained from a theoretical horizontally polarized MEXH data set, in the vertical (110) plane. (c) As in (b), but corrected for the effects of the polarization of the incident radiation.

images in the outlying planes of Fig. 12 are strongly suppressed in Fig. 14(b), both due to their distance from the Ge emitter, and the vertical polarization of the incident radia-

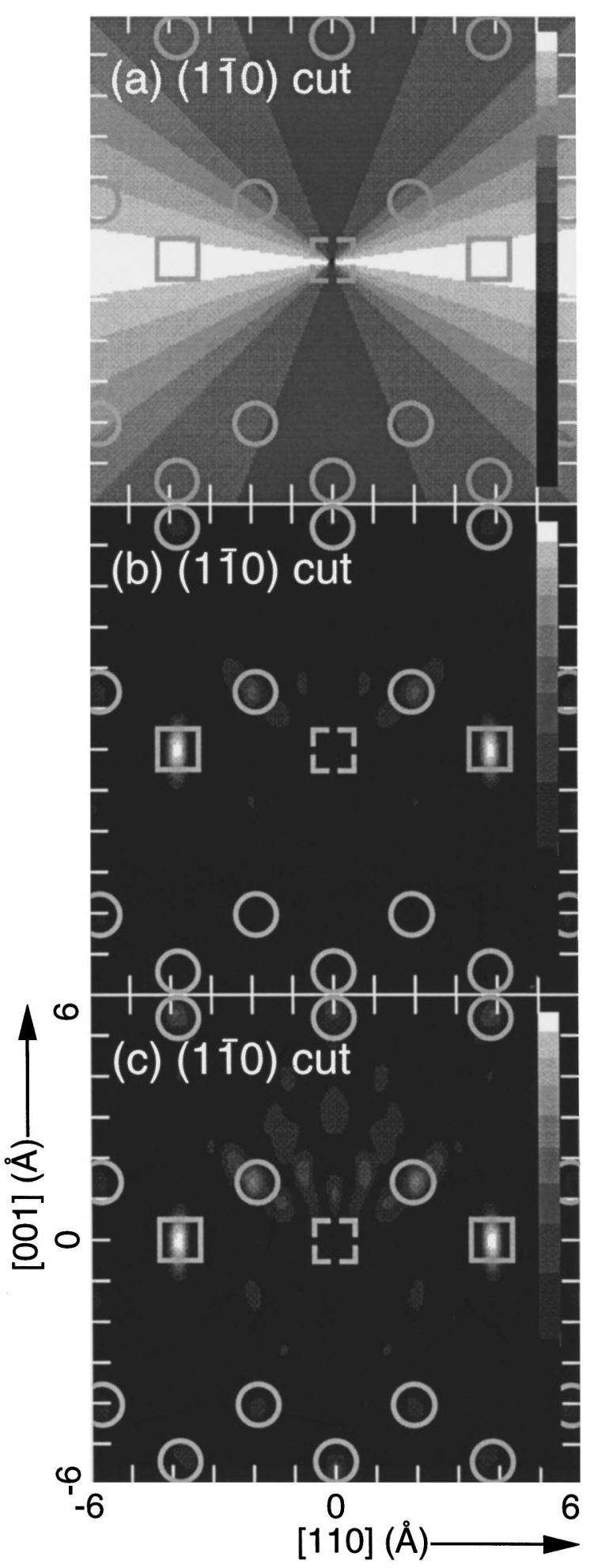

FIG. 14. As in Fig. 13, but for the case of vertically polarized incident radiation.

tion. Thus, recording MEXH intensities with vertically polarized incident radiation would not be most ideal for this particular system, but would be ideal for the imaging of other structures where atomic images in the horizontal (001) plane would be of more interest.

Also, note, the presence of faint image aberrations in Figs. 13(b) and 14(b) near the emitter, as compared to Fig. 12. These aberrations are due to there being less holographic 
information overall (i.e., fewer visible hologram fringes) in the MEXH $\chi(\mathbf{k})$ data sets obtained with polarized incident radiation, relative to the set obtained with unpolarized incident radiation (cf. Figs. 6-11).

The relative intensities of the atomic sites that have been preferentially/detrimentally imaged due to the polarization of the incident radiation can be restored. The use of a so-called SWIFT (scattered-wave included fourier transform) correction to the reconstruction algorithm of Eq. (1) is one method for accomplishing this, given by ${ }^{4(a)}$

$$
U\left(\mathbf{r}^{\prime}\right) \equiv \iiint_{K} \mathbf{d}^{3} \mathbf{k} \cdot \frac{e^{-i\left(\mathbf{k} \cdot \mathbf{r}^{\prime}-k r^{\prime}\right)} \chi(\mathbf{k})}{f_{\mathrm{Fe}}\left(\Theta_{\mathbf{k}}^{\mathbf{r}^{\prime}}\right) \sin \left(\Theta_{\hat{\mathbf{e}}}^{\mathbf{r}^{\prime}}\right)} .
$$

Here, one is simply dividing by the strength of the scattered wave associated with an atom along $\mathbf{r}^{\prime}$. However, doing this type of correction becomes problematic due to the nodes of the Thomson scattering factor $\sin \left(\Theta_{\hat{e}}^{\mathbf{r}^{\prime}}\right)$, such that the integrand in Eq. (3) will have divisions by zero. An ad hoc solution would then be to limit the SWIFT correction to the angular regions where the Thomson scattering factor is appreciably nonzero. ${ }^{27,28}$ However, we also note that the relative intensities of atomic sites enhanced/diminished by polarization effects can be restored simply by dividing the images by the appropriate enhancement ratios $R_{\text {enh }}$ $=U_{\text {polarized }}\left(\mathbf{r}^{\prime}\right) / U_{\text {unpolarized }}\left(\mathbf{r}^{\prime}\right)$. Determining this ratio does not require the direct experimental measurement of $\chi(\mathbf{k})$ intensities obtained using polarized and unpolarized incident radiation, as we find from an analysis of exact calculations for various geometries that it can be approximated using

$$
R_{\text {enh }}=\frac{U_{\text {polarized }}\left(\mathbf{r}^{\prime}\right)}{U_{\text {unpolarized }}\left(\mathbf{r}^{\prime}\right)} \approx \frac{\iiint_{k} \mathbf{d}^{3} \mathbf{k} \cdot \sin \left(\Theta_{\hat{\mathbf{e}}}^{\mathbf{k}^{\prime} \equiv \mathbf{r}^{\prime}}\right)}{\iiint_{k} \mathbf{d}^{3} \mathbf{k} \cdot \frac{1}{2} \sqrt{1+\cos ^{2}\left(\Theta_{\mathbf{k}}^{\mathbf{k}^{\prime} \equiv \mathbf{r}^{\prime}}\right)}} .
$$

The enhancement ratio is thus approximated by the relative contribution to an atomic image at $\mathbf{r}^{\prime}$ due to the $\mathbf{k}$-space domains in $\chi(\mathbf{k})$ that are selectively enhanced by the polarized Thomson scattering factor. Figures 13(c) and 14(c) show the reconstructed atomic images of Figs. 13(b) and 14(b) that have been corrected by dividing out the appropriate approximate polarization (horizontal and vertical, respectively) enhancement factor of Eq. (4). The relative intensities between all of the atomic sites in the corrected images of Figs. 13(c) and 14(c) are now well restored to uniform values, in comparison to the unpolarized atomic images of Fig. 12. Note that the atomic sites that have been detrimentally affected by the use of polarized incident radiation are discernible in both uncorrected [Figs. 13(b) and 14(b)] and corrected images [Figs. 13(c) and 14(c)]; merely the relative intensities between all atomic sites have been equalized in the corrected polarized images. As expected, the faint image aberrations in Figs. 13(b) and 14(b) persist in the corrected images of Figs. 13(c) and 14(c), due to the overall loss of signal to noise in polarized incident-radiation $\chi(\mathbf{k})$ data sets, compared to the image reconstructed from the unpolarized incident-radiation $\chi(\mathbf{k})$ data set (Fig. 12).

\section{CONCLUSIONS}

Multiple energy x-ray holography holds much promise in the imaging of local atomic structure surrounding a specific emitter species of interest, as demonstrated here in the first experimental data obtained with it, and for the model system of buried atomic $\delta$ layers. However, there are strong effects in these atomic images due to the Thomson scattering cross section when the linearly polarized radiation is used for exciting the fluorescence signal. Utilization of horizontally or vertically polarized incident radiation can emphasize vertical or horizontal atomic structures, respectively. Approximate procedures for correcting for this nonuniformity in image strengths are also discussed. If full three-dimensional atomic structural information is desired from a specific sample, then either unpolarized or circularly polarized radiation should be used for excitation, or the sample should be mounted such that it can be illuminated by either linear polarization mode relative to the polar and azimuthal rotations being carried out on it. MEXH intensities that would be measured for the case of unpolarized incident radiation also can be determined from the two individual polarized MEXH data sets as obtained over most of the solid angle above the sample.

\section{ACKNOWLEDGMENTS}

Research at UC Davis was supported in part by the Office of Naval Research (Contract Nos. N00014-90-5-1457 and N00014-94-1-0162), by the Director, Office of Energy Research, Office of Basic Energy Sciences, Material Sciences Division of the U.S. Department of Energy [Contract No. DE-AC03-76SF00098], the National Energy Research Supercomputer Center, and by the International Centre for Diffraction Data. Research at Oak Ridge National Laboratory beamline X-14A at the National Synchrotron Light Source, Brookhaven National Laboratory, was supported by the Division of Material Sciences and Division of Chemical Sciences of the U.S. Department of Energy, and by Lockheed Martin Energy Systems, Inc. (Contract No. DE-AC05840R21400).
*Present address: Advanced Photon Source at Argonne National Laboratory, Argonne, IL, 60439.

${ }^{1}$ D. Gabor, Nature (London) 161, 777 (1948).

${ }^{2}$ A. Szöke, in Short Wavelength Coherent Radiation: Generation and Applications, edited by T. Attwood and J. Boker, AIP Conf. Proc. No. 147 (AIP, New York, 1986), p. 361.

${ }^{3}$ (a) J. J. Barton, Phys. Rev. Lett. 61, 1356 (1988); 67, 3106 (1991). (b) J. J. Barton and L. J. Terminello, paper presented at the Third International Conference on the Structure of Surfaces,
Milwaukee, July 1990, and in Structure of Surfaces III, edited by S. Y. Tong, M. A. Van Hove, X. Xide, and K. Takanayagi (Springer-Verlag, Berlin, 1991), p. 107. (c) S. Y. Tong, H. Huang, and C. M. Wei, Phys. Rev. B 46, 2452 (1992), and references therein.

4 (a) B. P. Tonner, Z.-L. Han, G. R. Harp, and D. K. Saldin, Phys. Rev. B 43, 14423 (1991). (b) D. K. Saldin, K. Reuter, P. L. De Andres, H. Wedler, X. Chen, J. B. Pendry, and K. Heinz, ibid. 54, 8172 (1996). 
${ }^{5}$ (a) S. Y. Tong, H. Li, and H. Huang, Phys. Rev. B 51, 1850 (1995). (b) H. Wu and G. J. Lapeyre, ibid. 51, 14549 (1995). (c) J. M. Roesler, M. T. Sieger, T.-C. Chiang, Surf. Sci. 329, L588 (1995), and references therein.

${ }^{6}$ P. J. Rous and M. H. Rubin, Surf. Sci. 316, L1068 (1994).

${ }^{7}$ P. Hofmann, K.-M. Schindler, V. Fritzsche, S. Bao, A. M. Bradshaw, and D. P. Woodruff, J. Vac. Sci. Technol. A 12, 2045 (1994); Surf. Sci. 337, 169 (1995), and references therein.

${ }^{8}$ (a) L. J. Terminello, B. L. Petersen, and J. J. Barton, J. Electron Spectrosc. Relat. Phenom. 75, 229 (1995), and references therein. (b) H. Wu, G. J. Lapeyre, H. Huang, and S. Y. Tong, Phys. Rev. Lett. 71, 251 (1993), and references therein. (c) M. Zharnikov, M. Weinelt, P. Zebisch, M. Stichler, and H.-P. Steinrück, Surf. Sci. 334, 114 (1995), and references therein. (d) R. Denecke, R. Eckstein, L. Ley, A. E. Bocquet, J. D. Riley, and R. C. G. Leckey, ibid. 331-333, 1085 (1995). (e) J. G. Tobin, G. D. Waddill, H. Hi, and S. Y. Tong, ibid. 334, 263 (1995), and references therein.

${ }^{9}$ (a) H. Li, S. Y. Tong, D. Naumovic, A. Stuck, and J. Osterwalder, Phys. Rev. B 47, 10036 (1993). (b) D. K. Saldin, G. R. Harp, and X. Chen, ibid. 48, 8234 (1993), and references therein.

${ }^{10}$ (a) Z.-L. Han, S. Hardcastle, G. R. Harp, H. Li, X.-D. Wang, J. Zhang, and B. P. Tonner, Surf. Sci. 258, 313 (1991), and references therein. (b) I. H. Hong, S. C. Shyu, Y. C. Chou, and C. M. Wei, Phys. Rev. B 52, 16884 (1995), and references therein.

${ }^{11}$ (a) M. A. Mendez, C. Glück, J. Guerrero, P. L. Andres, K. Heinz, D. K. Saldin, and J. B. Pendry, Phys. Rev. B 45, 9402 (1992), and references therein. (b) C. M. Wei, S. Y. Tong, H. Wedler, M. A. Mendez, and K. Heinz, Phys. Rev. Lett. 72, 2434 (1994), and references therein.

${ }^{12}$ S. Y. Tong, H. Huang, and X. Q. Guo, Phys. Rev. Lett. 69, 3654 (1992).
${ }^{13}$ M. Tegze and G. Faigel, Europhys. Lett. 16, 41 (1991).

${ }^{14}$ P. M. Len, S. Thevuthasan, C. S. Fadley, A. P. Kaduwela, and M. A. Van Hove, Phys. Rev. B 50, 11275 (1994).

${ }^{15}$ M. Tegze and G. Faigel, Nature (London) 380, 49 (1996).

${ }^{16}$ T. Gog, P. M. Len, G. Materlik, D. Bahr, C. Sanchez-Hanke, and C. S. Fadley, Phys. Rev. Lett. 76, 3132 (1996).

${ }^{17}$ T. Gog, R.-H. Menke, F. Arfelli, P. M. Len, C. S. Fadley, and G. Materlik, Synch. Rad. News 9, 30 (1996).

${ }^{18}$ P. M. Len, T. Gog, C. S. Fadley, and G. Materlik, Phys. Rev. B 55, 3323 (1997).

${ }^{19}$ P. M. Len, C. S. Fadley, and G. Materlik, in X-ray and InnerShell Processes: 17th International Conference, edited by R. L. Johnson, H. Schmidt-Boeckering, and B. F. Sonntag, AIP Conf. Proc. No. 389 (AIP, New York, 1997), p. 295.

${ }^{20}$ E. Rotenberg and J. D. Denlinger (private communication).

${ }^{21}$ D. K. Saldin, G. R. Harp, B. L. Chen, and B. P. Tonner, Phys. Rev. B 44, 2480 (1991).

${ }^{22}$ S. Thevuthasan, G. S. Herman, A. P. Kaduwela, T. T. Tran, Y. J. Kim, R. S. Saiki, and C. S. Fadley, J. Vac. Sci. Technol. A 10, 2261 (1992).

${ }^{23}$ P. Hu and D. A. King, Phys. Rev. B 46, 13615 (1992).

${ }^{24}$ G. R. Harp, D. K. Saldin, X. Chen, Z.-L. Han, and B. P. Tonner, J. Electron Spectrosc. Relat. Phenom. 57, 331 (1991).

${ }^{25}$ International Tables for X-ray Crystallography, edited by $\mathrm{K}$. Lonsdale (Reidel, Dordrecht, 1968), Vol. III.

${ }^{26}$ J. Falta, T. Gog, G. Materlik, B. H. Muller, and M. Horn-von Hoegen, Phys. Rev. B 51, 7598 (1995), and references therein.

${ }^{27}$ S. Thevuthasan, G. S. Herman, A. P. Kaduwela, T. T. Tran, Y. J. Kim, R. S. Saiki, and C. S. Fadley, J. Vac. Sci. Technol. A 10, 2261 (1992).

${ }^{28}$ J. Osterwalder, R. Fasel, A. Stück, P. Aebi, and L. Schlapbach, J. Electron Spectrosc. Relat. Phenom. 68, 1 (1994). 